www.nature.com/clinicalpractice/rheum

A random sample of 100 radiographs was assessed, from individuals awaiting total knee replacement of the affected joint(s) at a single UK center. These were compared with 75 radiographs from individuals who had chronic knee pain but were not awaiting knee replacement, and 75 individuals who had hip pain, but no knee pain, during the previous year. Small, medium and large templates were developed to accommodate differently sized knees. Bone attrition (score $\geq 1$ ) was found in 59 radiographs from individuals awaiting surgery, compared with 7 radiographs from individuals not awaiting surgery and 1 radiograph from an asymptomatic individual.

Interobserver and intraobserver reliability for bone attrition scores were high. Bone attrition scores did not correlate well with other radiologic features of osteoarthritis and did not show a 'ceiling' effect in more advanced disease; therefore, the authors say that bone attrition scores provide a distinct measurement of the severity of knee osteoarthritis. They suggest that this scheme might be most useful for classifying severe knee osteoarthritis, where schemes based on other radiographic features of the disease lack discrimination.

Caroline Barranco

Original article Dieppe PA et al. (2005) Assessing bone loss on radiographs of the knee in osteoarthritis: a cross-sectional study. Arthritis Rheum 52: 3536-3541

\section{Rheumatoid arthritis and TNF antagonists as risk factors for skin cancer}

A large US cohort study has indicated that patients with rheumatoid arthritis (RA) have an increased risk of developing non-melanoma skin cancer (NMSC) compared with osteoarthritis (OA) patients, and that tumor necrosis factor (TNF) inhibitors could also be a risk factor for NMSC in these patients. This is consistent with results from European studies, and validates concern about reports of NMSC after anti-TNF therapy. In the current study, Chakravarty et al. aimed to determine the incidence rate of NMSC in RA patients, to identify possible risk factors, and to assess the role of immunosuppressive treatment in NMSC development.

Data from 15,789 RA patients and 3,639 OA patients were available for analysis in this trial. The crude incidence rate of NMSC was 18.1 and 20.4 per 1,000 person-years for RA and OA patients respectively, but, after adjusting for covariates, RA was associated with an increased risk of NMSC compared with OA (hazard ratio $=1.19$ ). There was a slight (but not significant) increased risk of NMSC in patients receiving anti-TNF alone, and a twofold risk of developing NMSC in patients receiving methotrexate and anti-TNF. The study also found that older age, male sex and Caucasian ancestry were risk factors for NSMC.

The trial had several limitations: some diagnoses of NMSC were not validated; NSMC diagnoses were not divided into subsets; and not all potential causes of NMSC were measured. Further trials to study the effect of NMSC subsets, and to define the associations between RA and NMSC are required. The authors conclude that skin cancer screening in RA patients, and particularly those receiving immunosuppressive therapy, could be warranted.

Rachel Murphy

Original article Chakravarty EF et al. (2005) Skin cancer, rheumatoid arthritis, and tumor necrosis factor inhibitors. J Rheumatol 32: 2130-2135

\section{'Molecular surgery' in the treatment of rheumatoid arthritis}

Rheumatoid arthritis (RA) is characterized by a hyperplastic synovial membrane that secretes an array of inflammatory molecules, which leads to the destruction of cartilage and bone. Surgical synovectomy can temporarily ameliorate this process but causes trauma to surrounding tissues. 'Molecular surgery', by initiation of apoptosis in synoviocytes via activation of the membrane-bound death receptor Fas, is being explored as a potential new strategy for eliminating hyperplastic tissue.

Zhang et al. tested this strategy by subcutaneously grafting synovium and cartilage from patients with RA into the backs of SEVERE COMBINED IMMUNODEFICIENT (SCID) MICE. The grafted tissue was injected five times at 2-weekly intervals with a recombinant adenovirus transfected with the gene for the Fas ligand (FASLG), a control adenovirus, or phosphate-buffered saline.

Synovial tissues were analyzed postmortem; compared with those of controls, the synovial tissues of mice injected with FASLG

\section{GLOSSARY}

SEVERE COMBINED IMMUNODEFICIENT (SCID) MICE

Mice with a spontaneous mutation in a gene

necessary for $\lg G$ and

T-cell receptor gene rearrangements that lack nearly all $T$ cells and $B$ cells 